Check for updates

Cite this: J. Mater. Chem. B, 2017, 5,6292

\title{
Surface enhancement of a molecularly imprinted polymer film using sacrificial silica beads for increasing L-arabitol chemosensor sensitivity and detectability
}

\author{
Marcin Dabrowski, ${ }^{a}$ Maciej Cieplak, ${ }^{a}$ Krzysztof Noworyta, (D) a Matthias Heim, ${ }^{b}$ \\ Witold Adamkiewicz, ${ }^{a}$ Alexander Kuhn, ${ }^{b}$ Piyush Sindhu Sharma (D) *a and \\ Wlodzimierz Kutner (D) *ac
}

\begin{abstract}
Molecular imprinting in polymers leads, among others, to synthetic receptors of high selectivity, comparable to that of their biological counterparts. Deposition of a thin non-porous molecularly imprinted polymer (MIP) film directly on a transducer surface enables fabrication of chemosensors for various health relevant biocompounds. However, the sensitivity of a chemosensor with such an MIP film as the recognition unit is limited, mostly because of slow analyte diffusion through this film. Herein, a simple procedure was developed to enhance, in a controlled way, the active surface area of an L-arabitol imprinted polymer film. For this, a macroporous MIP-(L-arabitol) film was synthesized and simultaneously deposited on a gold electrode of a quartz crystal resonator transducer by potentiodynamic electropolymerization. This large surface area film effectively enhanced analytical signals of mass changes at a quartz crystal microbalance. Hence, the L-arabitol limit of quantification was $\sim 16$-fold better than that of the corresponding non-porous MIP film of the same mass.
\end{abstract}

Received 23rd May 2017

Accepted 14th July 2017

DOI: $10.1039 / c 7 t b 01407 d$

rsc.li/materials-b

\section{Introduction}

Development of robust and sensitive sensors for real-time analytical detection is important in clinical diagnosis. Generally, these sensors contain recognition units derived either from biological ${ }^{1,2}$ or chemically synthesized materials ${ }^{3,4}$ to provide the desired sensing selectivity. Depending on the recognition materials used, these sensors are categorized as bio- or chemosensors. Recognition units prepared from chemically synthesized materials are considered advantageous in comparison with biological ones. The major disadvantage associated with biosensors includes limited stability (because of poor tolerance of extreme solution acidity or basicity, temperature, some organic solvents, and exposure to external fields, e.g., an electromagnetic or ultrasonic field), short lifetime, low availability, inadequate reproducibility, and high cost because of the necessity of tedious purification. Moreover, different receptor orientations on the transducer surface can affect the performance of biosensors. In contrast, chemical sensors appear more robust and reproducible. In these

\footnotetext{
${ }^{a}$ Institute of Physical Chemistry, Polish Academy of Sciences, Kasprzaka 44/52, 01-224 Warsaw, Poland. E-mail: psharma@ichf.edu.pl,wkutner@ichf.edu.pl

${ }^{b}$ Univ. Bordeaux, CNRS, ISM, ENSCBP, 16 Avenue Pey Berland, 33607 Pessac, France

${ }^{c}$ Faculty of Mathematics and Natural Sciences, School of Sciences, Cardinal Stefan Wyszynski University in Warsaw, Woycickiego 1/3, 01-815 Warsaw, Poland
}

chemosensors, selective analyte determination is attained by the deposition of synthetic receptors directly on the transducer surface. A lot of effort has been focused on designing and synthesizing new materials as alternatives to biological counterparts. $^{5-7}$

One of such research approaches involves imprinting of analytes in polymers. ${ }^{7-9}$ In this approach, synthetic materials are prepared by copolymerization of functional and cross-linking monomers in the presence of a template in a porogenic solvent. During this synthesis, first, the monomers bearing suitable functionalities and the template molecules are pre-organized in solution to form a complex via either noncovalent or covalent interactions between their complementary binding functionalities. The structures of these complexes are then "frozen" with a cross-linking monomer to result in a molecularly imprinted polymer (MIP) matrix. After subsequent template removal, vacant molecular cavities, complementary in their size and shape to the template molecule, are left in this matrix. These cavities are then capable of selective binding of analyte molecules, even in the presence of similarly structured molecules of interferenes.

MIP preparation as a thin non-porous film, directly deposited on the transducer surface, is the most common way to integrate receptors for devising a chemosensor. Towards that, different strategies have already been developed. ${ }^{10}$ However, mass transport of analytes in non-porous films is hindered because of slow diffusion to less accessible imprinted cavities that are buried 
deeply inside the MIP film. This mass transport hindrance severely limits the sensitivity of MIP chemosensors. Therefore, several attempts have been made to enhance this transport. One possible solution involves the enhancement of the MIP film active surface area. ${ }^{11}$ This can easily be achieved by preparing an MIP as the porous film. One approach, which was proposed recently to increase this area, is based on growing the MIP film in between self-assembled nanostructures, e.g., colloidal silica or spherical polymer beads, ${ }^{11-13}$ similarly to what has been reported for metal films with controlled porosity. ${ }^{14-17}$ In addition to a large (surface area)-to-volume ratio, well-defined and uniform macroporous structures were produced that way. ${ }^{11}$ The resulting films were capable of binding analytes more effectively than the nonporous MIP films. ${ }^{12,13,18}$

Clinical statistics shows that the Candida yeasts ${ }^{19,20}$ have been responsible for the growing number of invasive fungal infections in recent years. ${ }^{21}$ Despite the introduction of novel antifungal therapeutic treatments, the rate of mortality caused by these infections is still very high. ${ }^{21}$ Early diagnosis of these infections seems to be crucial for successful therapy. ${ }^{22}$ Now, there is no adequate method in terms of sensitivity and selectivity for the diagnosis of invasive candidiasis in humans. Results from blood and tissue cultures are still considered the 'gold standard', although a number of non-culture methods have been developed including antibody and antigen tests, DNA amplification tests, and detection of Candida metabolites.

A method that has gained considerable attention in recent years involves the determination of $\mathrm{D}$-arabitol in body fluids. This polyol is produced in vitro by most pathogenic and medically important Candida sp. ${ }^{23,24}$ Moreover, it is produced by the human organism in an equimolar ratio with the L-enantiomeric form. Physiological concentrations of $\mathrm{D}^{-}$and $\mathrm{L}$-arabitol in blood plasma and urine are in the micromolar range $\mathrm{e}^{25}$ and their abnormal concentrations may indicate the Candida sp. infection. However, the concentration of D-arabitol in body fluids can be affected by many other factors of patients' variability, e.g., renal function. This effect lowers the accuracy of the diagnosis. To overcome this deficiency, two strategies were developed. Both of them rely on referencing the $\mathrm{D}$-arabitol concentration to concentrations of some internal standards, which indicate the efficiency of renal function. These two standards are L-arabitol and creatinine. Therefore, more accurate methods of diagnosis of infections caused by Candida sp. involve the determination of the (D-arabitol)-to-creatinine ratio, or the (D-)-to-(L-arabitol) ratio in body fluids. ${ }^{26,27}$

In our previous effort to elaborate chemosensors for the above polyols, we have designed synthetic receptors for D-arabitol and integrated them with suitable transducers. ${ }^{28}$ However, the sensitivity and limit of detection (LOD) of these former chemosensors were insufficient for arabitol determination in blood plasma samples. Therefore, there is a clear need to develop a sugar-alcohol templated MIP film with superior sensitivity. Moreover, it is reasonable to target the less abundant L-enantiomer to obtain an enantioselective chemosensor.

For this reason, herein, we explored the use of colloidal crystals based on silica beads as sacrificial nanostencils for electrodeposition of macroporous MIP-(L-arabitol) films with an inverse opal structure. The obtained films were then used as recognition units in piezoelectric microgravimetry (PM) measurements at an electrochemical quartz crystal microbalance, EQCM. Undoubtedly, the major advantage of this synergistic combination of methods is the ability to determine L-arabitol at concentrations much lower than those attained with a non-porous MIP film. Hence, this decreased LOD should enable early and immediate initiation of treatment and protection of a patient, thus avoiding unnecessary complications. ${ }^{21,29}$ Therefore, monitoring the health status of patients under antifungal treatment should become relatively straightforward. ${ }^{30}$

\section{Materials and methods}

\section{Chemicals}

2,2'-Bithiophene-boronic acid and 3,3'-bithiophene were purchased from Combi-Blocks, while acetonitrile, 3-aminopropyltriethoxysilane, tetraethoxysilane, and tetrabutylammonium perchlorate were from Sigma-Aldrich. L-Arabitol, D-arabitol, xylitol, and ribitol were bought from Alfa-Aesar. Ethanol, sodium hydroxide, and hydrochloric acid were supplied by POCH. Potassium hexacyanoferrate(II) was from CHEMPUR. Milli-Q water with 18.2 $\mathrm{M} \Omega \mathrm{cm}$ resistivity was used for all of the Langmuir and Langmuir-Blodgett (LB) experiments.

\section{Apparatus}

An AUTOLAB electrochemistry system (Metrohm Autolab), equipped with a PGSTAT12 potentiostat and a FRA2 frequency response analyzer, was used for the potentiodynamic and differential pulse voltammetry (DPV) experiments. A threeelectrode one-compartment V-shaped glass minicell was used for the electrochemical experiments. A $1 \mathrm{~mm}$ diameter Pt disk electrode, a saturated $\mathrm{Ag} \mid \mathrm{AgCl}$ electrode, and Pt foil were used as the working, reference, and auxiliary electrode, respectively.

Model EQCM 5610 and EQCM 5710 quartz crystal microbalances, controlled by the EQCM 5710-S2 software, all of the Institute of Physical Chemistry (Warsaw, Poland), were used to perform PM experiments under flow-injection analysis (FIA) and batch analysis conditions, respectively. The resonance frequency change was measured with $1 \mathrm{~Hz}$ resolution using $14 \mathrm{~mm}$ diameter, AT-cut, plano-plano, unpolished gold-coated quartz crystal resonators, Au-QCRs ${ }^{31}$ (relative surface area, $R_{\mathrm{Sa}}=2.0$ ), of $10 \mathrm{MHz}$ resonance frequency with $5 \mathrm{~mm}$ diameter and $100 \mathrm{~nm}$ thick Au film electrodes evaporated over Ti underlayer films on both resonator sides. However, only one side was wetted by the working solution and the $\mathrm{Au}$ film electrode of this side was used both as the working electrode and the substrate for the MIP film. Then, the Au-QCRs were rinsed with methanol, next with acetone, and then dried in air. Pt coil and Ag wire were used as the counter and pseudoreference electrode, respectively.

A Zepto model plasma cleaner of $100 \mathrm{~W}$ power from Diener Electronic was used for cleaning and hydrophilizing the surfaces of the Au-QCR. A rectangular $(7 \times 75) \mathrm{cm}^{2}$ Langmuir trough type BAM 601 with an accessory from Nima Technology, controlled by NIMA TR620 software, was used to perform the Langmuir 
experiments and LB transfer of silica nanobeads onto the hydrophilized surfaces of Au-QCRs. A MPW Medical Instruments centrifuge model MPW-351R with the rotor no. 11457 was used for the centrifugation of nanobead suspensions for the Langmuir and LB experiments. The MIP films were imaged with scanning electron microscopy (SEM) using a Nova NanoSEM 450 microscope of FEI Nova.

\section{Chemosensor fabrication}

Silica colloidal crystals. For silica colloidal crystal preparation, first, silica nanobeads were synthesized, and then Langmuir films of these nanobeads were prepared, and subsequently LB transferred onto Au-QCRs. Silica nanobeads of $\sim 330 \mathrm{~nm}$ diameter were synthesized according to the batch or the semicontinuous procedure inspired by the Stöber sol-gel process. ${ }^{32}$ These nanobeads were surface modified with 3-aminopropyltriethoxysilane, and then washed, as described previously. ${ }^{33-35}$

Purified and dehydrated nanobeads were suspended in a mixed solvent solution of anhydrous ethanol and chloroform $(1: 4, v: v)$ with the nanobead concentration of $\sim 10 \mathrm{mg} \mathrm{mL}^{-1}$. Then, the resulting suspension was spread over the air-water interface of the Langmuir trough filled with Milli-Q water. After organic solvent evaporation, the nanobeads present at the air-water interface were compressed with a barrier speed of $10 \mathrm{~cm}^{2} \mathrm{~min}^{-1}$ to the target LB transfer pressure of $8.0 \mathrm{mN} \mathrm{m}^{-1} .36$

The Au-QCRs were rinsed, consecutively, with water, methanol, and acetone. Subsequently, they were treated with $50 \mathrm{~W}$ oxygen plasma under the oxygen pressure of $0.25 \mathrm{kPa}$ for $10 \mathrm{~min}$. A freshly plasma cleaned Au-QCR was used as the substrate for the LB deposition of the colloidal nanobead crystals. This substrate was immersed in the trough liquid, and then withdrawn four times, at the speed of $38 \mathrm{~mm} \mathrm{~min}^{-1}$ (the maximum speed available) and $1 \mathrm{~mm} \mathrm{m^{-1 }}$, respectively. After the first withdrawal, the sample was dried and heated to promote adhesion of the nanobeads of the first layer to the Au electrode surface.

Deposition of MIP and NIP films on Au electrodes of quartz crystal resonators. Simultaneous synthesis and deposition of a MIP film onto the Au-QCR surface was performed by electropolymerization under potentiodynamic conditions similar to those described in previous reports. ${ }^{37}$ Both Au-QCRs with bare $\mathrm{Au}$ electrodes and the Au electrodes coated by four layers of silica beads, forming a colloidal crystal, were used for this purpose.

An EQCM stagnant solution system, comprising an EP-21 potentiostat of ELPAN, was used for the deposition with two potential cycles from 0.50 to $1.20 \mathrm{~V}$ vs. an $\mathrm{Ag} \mid \mathrm{AgCl}$ pseudo reference electrode at $100 \mathrm{mV} \mathrm{s}^{-1}$ scan rate. An acetonitrile solution of $1 \mathrm{mM}$ L-arabitol (template), $2 \mathrm{mM}$ 2,2'-bithiophene

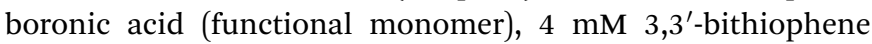
(cross-linking monomer), and $0.1 \mathrm{M}$ (TBA)ClO $\mathrm{Cl}_{4}$ (supporting electrolyte) was used for the electrochemical syntheses of MIP films. After electropolymerization, the MIP films were rinsed with acetonitrile to remove unreacted substrates and the electrolyte, and then dried in air.

A non-imprinted polymer (NIP) control film was deposited from the template-free solution using the same electropolymerization procedure.
Silica nanobead removal from the MIP film. The MIP film, deposited into the colloidal crystal-coated Au-QCR, was immersed for $5 \mathrm{~min}$ in $1 \% \mathrm{HF}$ to dissolve the nanobeads, thus resulting in the final macroporous MIP film.

Template removal from the MIP film. For the L-arabitol template extraction, the MIP films were immersed in $0.1 \mathrm{M}$ $\mathrm{HCl}$ for $2 \mathrm{~h}$ at $40{ }^{\circ} \mathrm{C}$. Then, the DPV curves were recorded with a solution of $10 \mathrm{mM} \mathrm{K}_{4}\left[\mathrm{Fe}(\mathrm{CN})_{6}\right]$ in $0.1 \mathrm{M} \mathrm{KCl}$ for different extraction times. During the DPV measurements, the potential range, potential step, pulse amplitude, and pulse duration were 0 to $0.50 \mathrm{~V}, 5 \mathrm{mV}, 25 \mathrm{mV}$, and $50 \mathrm{~ms}$, respectively. Moreover, the DPV curves were recorded for the NIP films before and after similar treatment.

Microscopic imaging of the macroporous MIP, non-porous MIP, and non-porous NIP films. The non-porous MIP and NIP films as well as macroporous MIP films were imaged with SEM using a $2.50 \mathrm{kV}$ accelerated electron beam. Moreover, the colloidal silica crystal assembled over the Au-QCR was imaged for the confirmation of the number of nanobead layers deposited by the LB transfer.

Analytical performance of the chemosensor under FIA conditions. For the determination of the analytical parameters of the fabricated chemosensor, a FIA system composed of a flow-through EQCM 5610 holder of IPC PAS, ${ }^{38}$ a Model KDS100 syringe pump of KD Scientific, and a Model 7725i rotary six-port valve of Rheodyne was used. Distilled water, pumped with a $35 \mu \mathrm{L} \mathrm{min}{ }^{-1}$ flow rate, was used as the carrier liquid. $100 \mu \mathrm{L}$ samples of solutions of increasing concentrations of $\mathrm{L}^{-}$or D-arabitol, xylitol, or ribitol were injected for constructing calibration plots.

\section{Results and discussion}

The nature of interaction between a template and functional monomers determines the performance of the designed MIP. Moreover, the number of recognition sites in the MIP directly depends on the nature and extent of the monomer-template interactions in the pre-polymerization solution. ${ }^{39}$ For instance, if the pre-polymerization complex involves only non-covalent interactions, the pre-polymerization solution at equilibrium will be a mixture of functional monomers, and non-complexed, partially complexed, and fully complexed templates. This equilibrium can generate heterogeneous binding sites and, ultimately, decrease the efficiency of molecular imprinting. This deficiency can be avoided by promoting pre-polymerization complex formation through reversible covalent bonding.

Reversible covalent bonding of boronic acids with cis-1,2- or 1,3-diols leads to the formation of five- or six-membered cyclic esters, respectively. These interactions were proven to be sufficiently strong. Hence, binding saccharides at millimolar or sub-millimolar concentration levels was possible, thus allowing boronic-acid-based saccharide sensing in biologically relevant environments. ${ }^{6,40}$ Therefore, we used boronic acid derivatized functional monomers to imprint the L-arabitol polyol. Vicinal diols of arabitol were covalently bound by the $2,2^{\prime}$-bithiophene-5-boronic acid functional monomer for this imprinting. 


\section{Chemosensor fabrication}

Fabrication of colloidal crystals on Au electrodes of the quartz crystal resonators. The LB technique was used to transfer silica nanobeads onto the Au-QCR surface. A monolayer of silica nanobeads on the surface of the water subphase was compressed and the resulting compression isotherm is shown in Fig. 1. Its shape indicates that the monolayer is irreversibly destroyed (collapsed) at a surface pressure of $\sim 9 \mathrm{mN} \mathrm{m}^{-1}$. A constant high value of the isotherm slope between 0 and $8 \mathrm{mN} \mathrm{m}^{-1}$ indicates the absence of any phase transition in the monolayer. Thus, as expected, in this pressure range the monolayer can be transferred as a two-dimensional solid. This transfer was repeated four times to coat the $\mathrm{Au}$ electrodes of the Au-QCR with a four-layer film of closely packed silica nanobeads. It is desirable to fabricate a colloidal crystal composed of as many nanobead layers as possible. However, only four monolayers were deposited herein in order not to overload the Au-QCR with the deposited mass.

The SEM images (Fig. 2) confirmed the presence of four layers of silica nanobeads and their hexagonal-like packing, characteristic of a colloidal crystal. Although the structure of this crystal was imperfect, importantly, each nanobead was in contact with several other nanobeads. Fabrication of highly ordered colloidal crystals under our experimental conditions was difficult because the surface of the chemically unpolished Au-QCRs is relatively rough. ${ }^{31}$

Deposition of MIP and NIP films on Au electrodes of quartz crystal resonators. The non-porous MIP films were deposited on gold electrodes of Au-QCRs using potentiodynamic polymerization, as described in our previous work. ${ }^{28}$ The film deposition resulted in a decrease in resonance frequency by $\sim 4.0 \mathrm{kHz}$. To confirm the enhancement of the analytical signal by surface enhancement, an MIP film of similar mass was deposited on the Au-QCR coated with a colloidal crystal of silica nanobeads (Scheme 1). ${ }^{41}$ A sufficient amount of the 3,3'-bithiophene crosslinking monomer was added during polymerization, thus providing 3-D crosslinking to the polymer matrix and helping to maintain a suitable orientation of the recognition sites.

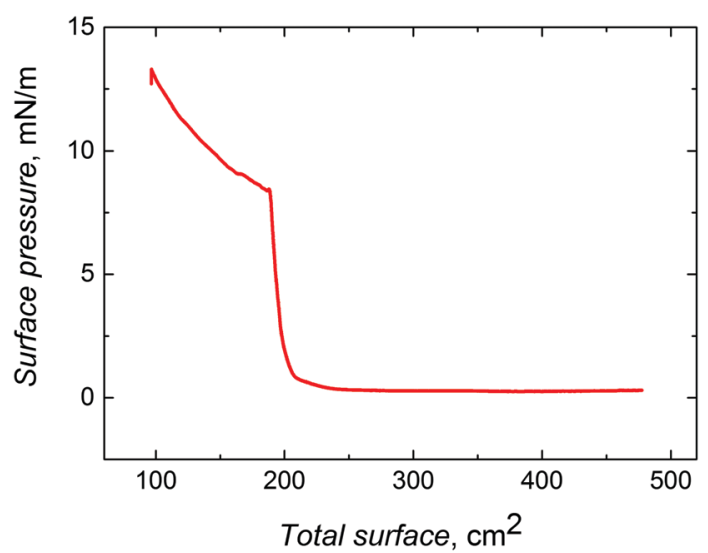

Fig. 1 Compression isotherm for $\sim 330 \mathrm{~nm}$ silica nanobeads, which were surface functionalized with 3-aminopropyltriethoxysilane. The barrier speed was $10 \mathrm{~cm}^{2} \mathrm{~min}^{-1}$.

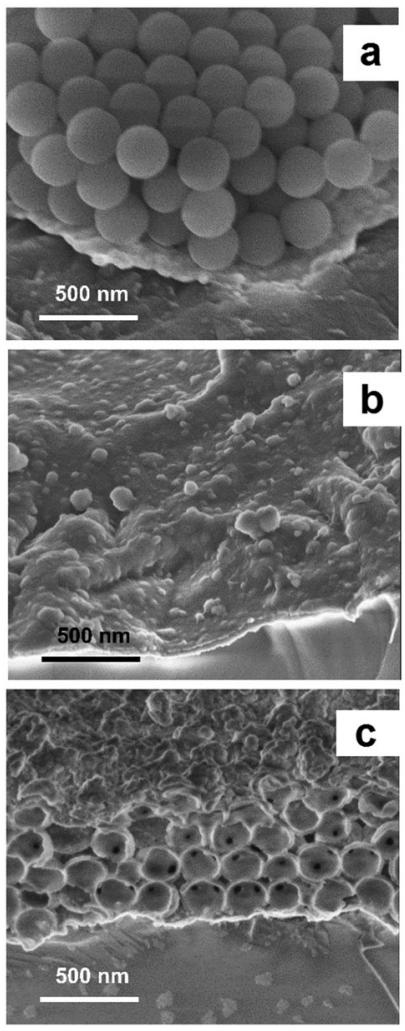

Fig. 2 The SEM images of side views of (a) a four-layer $330 \mathrm{~nm}$ diameter silica nanobead colloidal crystal, (b) a non-porous MIP film, and (c) a macroporous MIP film deposited on Au electrodes of quartz crystal resonators.

During potentiodynamic potential cycling, electro-oxidation at $\sim 1.1 \mathrm{~V}$ resulted in the generation of a bithiophene cation radical, which then initiated the polymerization. ${ }^{42} 3,3^{\prime}$-Bithiophene acts as an acceptor of the cation radical and, hence, passively participates in the electropolymerization. Therefore, the cross-linking monomer was added to the solution for electropolymerization at a much higher concentration than that of the functional monomer. The dynamic resistance change resulting from film deposition was relatively small, equaling $\sim 60 \Omega$, thus indicating a very low viscoelasticity change of the polymer film and that way showing that the films were rigid.

Template removal as well as electrochemical and microscopic characterization of the MIP and NIP films. The non-porous (L-arabitol)-templated MIP film was electrochemically characterized by DPV using the $\mathrm{Fe}(\mathrm{CN})_{6}{ }^{4-}$ redox probe before and after the extraction of the L-arabitol template (Fig. 3). This characterization was performed in order to test the probe permeation through the MIP film. The acid solution cleaves the $\mathrm{B}-\mathrm{O}-\mathrm{C}$ bonds and promotes the extraction of the L-arabitol template from the MIP. In contrast to DPV voltammograms of the MIP film before the extraction of the L-arabitol template (curve 1 in Fig. 3), the voltammograms for the film after the extraction for 1 and $1.5 \mathrm{~h}$ (curve 2 and 3, respectively, in Fig. 3) exhibited a very significant peak at $\sim 0.22 \mathrm{~V}$. This peak is characteristic of electroactivity of the redox probe. Permeation of the probe through the MIP film was faster after the extraction, thus indirectly confirming cleavage of the boronic ester bonds and extraction of 
a

b c d

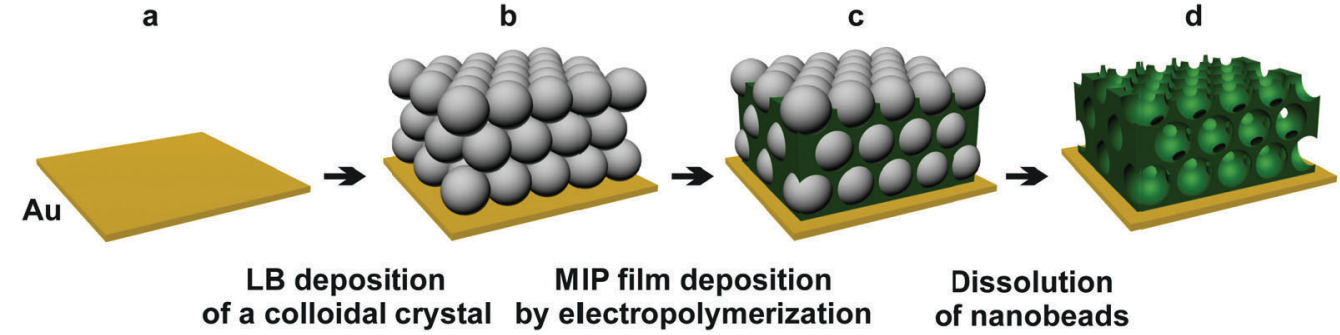

Scheme 1 Preparation of (a) the Au electrode, (b) the silica nanobead colloidal crystals by the LB technique, and then (c) deposition of the MIP by electropolymerization, followed by (d) the formation of the macroporous MIP film by dissolution of the silica beads.

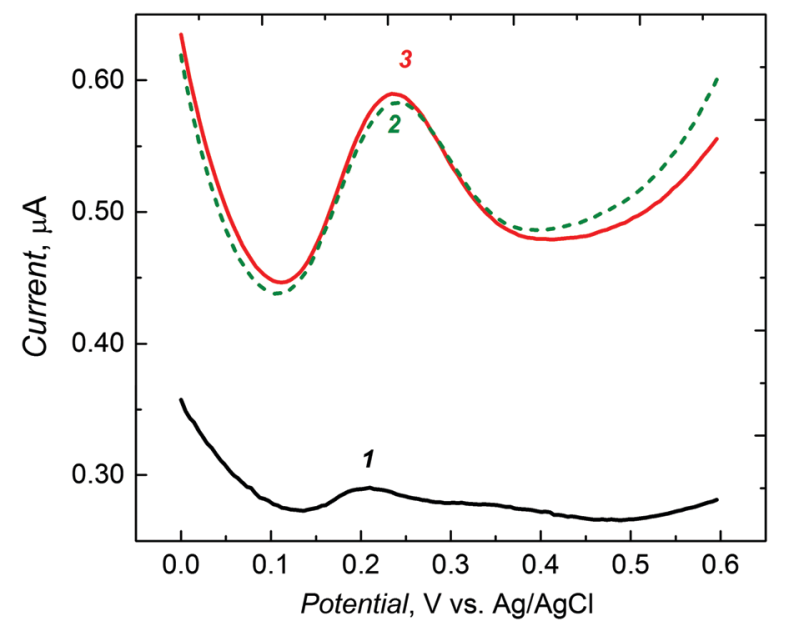

Fig. 3 Differential pulse voltammograms for $10 \mathrm{mM} \mathrm{K}_{4} \mathrm{Fe}(\mathrm{CN})_{6}$ in $0.1 \mathrm{M}$ $\mathrm{KCl}$ for a $1 \mathrm{~mm}$ diameter Pt disk electrode coated with the L-arabitol templated non-porous MIP film (1) before and after L-arabitol extraction with $0.1 \mathrm{M} \mathrm{HCl}$ for (2) $1 \mathrm{~h}$ and (3) $1.5 \mathrm{~h}$.

the $\mathrm{L}$-arabitol template, which resulted in emptying molecular cavities in the MIP.

The SEM imaging of MIP films allowed deeper insights into film morphology of the macroporous film structure (Fig. 2). Polythiophene deposited by electropolymerization does not form a typical inverse opal-like crystal structure (Scheme 1) but, instead, a structure composed of hollow spheres ${ }^{43}$ (Fig. 2c). Nevertheless, the 3-D structure of the macroporous MIP film obtained in this way was mechanically stable. The diameter of each pore in this structure was the same and close to $330 \mathrm{~nm}$. This corresponds to the diameter of the employed silica nanobeads. Moreover, most of the pores were interconnected, with small channels, with neighboring pores. Unfortunately, some pores of the outer polymer layer were closed (Fig. 2c). However, the pore closing film was relatively thin. Therefore, it only negligibly hindered permeation of the target analyte into the pores. Moreover, the specific surface area of the film composed of hollow spheres was higher than that of a typical inverse opal-like structure because of the presence of additional outer sides of the pore walls exposed to the solution. Therefore, introduction of macroporosity increased significantly the specific surface area of the polymer.

From the PM experiment, the density of the macroporous and non-porous MIP film was determined. The thickness of the non-porous MIP film was $200 \mathrm{~nm}$, whereas that of the macroporous film was $\sim 700 \mathrm{~nm}$. The mass of both MIPs (non-porous and macroporous) was the same $(3.47 \mu \mathrm{g})$. The calculated density (mass/volume) of the non-porous film was $\sim 890 \mathrm{~kg} \mathrm{~m}^{-3}$, whereas the apparent density of the macroporous MIP film was $\sim 3.5$ times lower $\left(\sim 247 \mathrm{~kg} \mathrm{~m}^{-3}\right)$.

Analytical performance of the MIP chemosensors under FIA conditions. After the extraction of the $\mathrm{L}$-arabitol template from the MIP films, the arabitol analyte and interference binding in MIPs was studied under FIA conditions to evaluate analytical parameters of the chemosensors. Nonporous MIP films of polythiophenes are rather rigid. ${ }^{44,45}$ Therefore, viscosity and density changes negligibly contribute to the measured resonant frequency changes. For such rigid films, the change of resonant frequency is opposite to the change of mass deposited on the resonator surface, as the Sauerbrey equation describes. ${ }^{44,46}$

When injected to the PM-FIA carrier liquid, the sample solution of L-arabitol flows over the (L-arabitol)-extracted MIP thin film, and then L-arabitol is temporarily captured by this film. This L-arabitol binding increases the mass of the MIP film and, hence, decreases the resonant frequency of the Au-QCR (curve 1 in Fig. 4a). When the concentration of the $\mathrm{L}$-arabitol in the carrier liquid decreases, it is eluted from the film with excess of the liquid and the resonant frequency increases back to its original baseline level. This reversible change in resonant frequency during L-arabitol binding and releasing confirms that the MIP film does not suffer from any detectable memory effects. Moreover, a series of injections for each calibration curve were repeated several times and the first sample of the following series was injected just after the last injection of the preceding one. Under these conditions, we also did not observe any memory effect.

The non-porous MIP film chemosensor determined L-arabitol in the concentration range of 0.15 to $1.25 \mathrm{mM}$ (curve 1 in Fig. 4a). Interestingly, when the non-porous MIP film coated Au-QCR was replaced with a macroporous MIP film coated Au-QCR in the FIA system, the chemosensor performance was enhanced appreciably (curve 2 in Fig. 4a). A 100\% sensitivity was gained for the macroporous MIP film compared to that of the non-porous MIP film (Table 1). This gain enabled the chemosensor to determine $\mathrm{L}$-arabitol at a concentration as low as $9 \mu \mathrm{M}$, i.e., $\sim 16$ times lower than that determined with the non-porous MIP film (0.15 mM). Apparently, this higher detectability was due to macroporosity of 

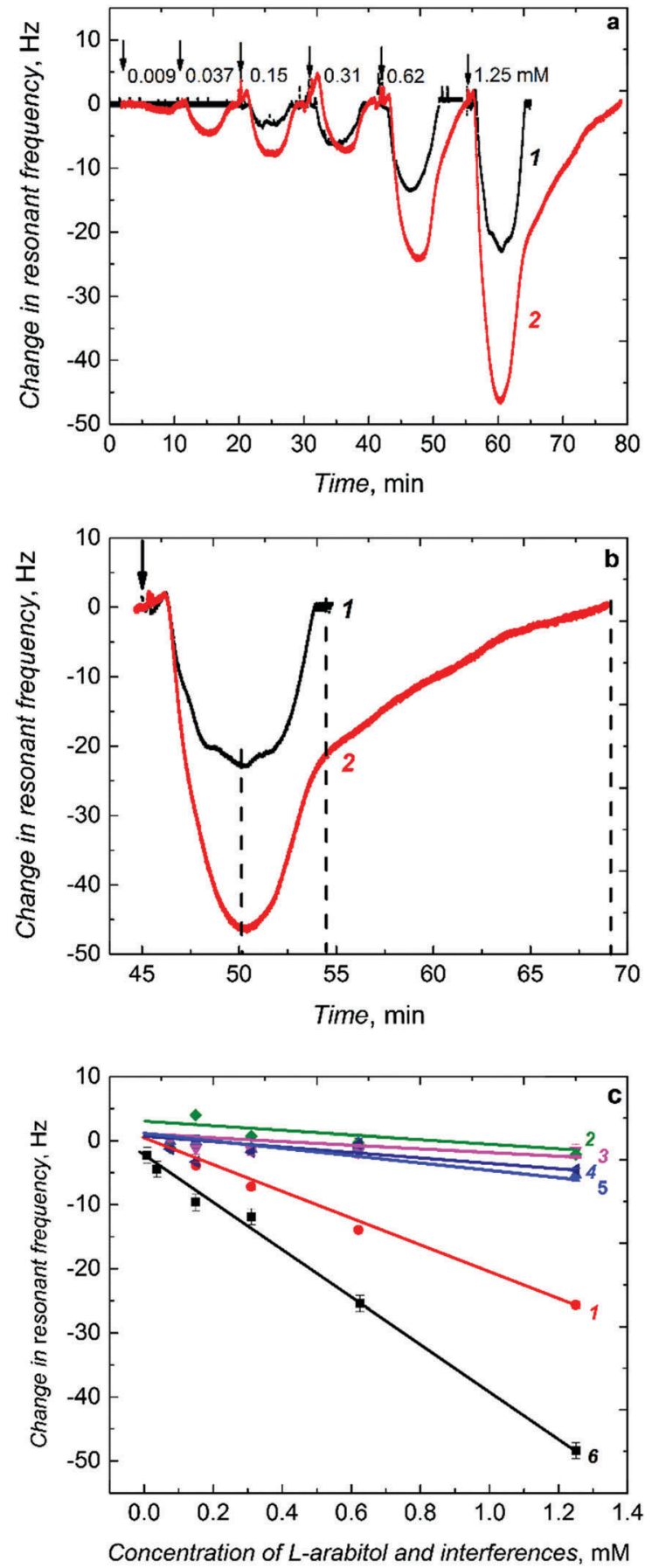

Fig. 4 (a) Time dependence of the resonant frequency change due to L-arabitol sample injection under FIA conditions for a piezoelectric microgravimetry chemosensor with the (1) non-porous and (2) macroporous (L-arabitol)-templated MIP film after L-arabitol extraction. (b) An expanded view of the $1.25 \mathrm{mM} \mathrm{L}$-arabitol injection over the (1) non-porous and (2) macroporous MIP film. (c) Calibration plots for the L-arabitol analyte ( 1 and 6$)$ and interferences (2 - D-arabitol, 3 - xylitol, and 4 - ribitol) for piezoelectric microgravimetry chemosensors with the non-porous MIP film $(1,2,3,4)$, the macroporous MIP film (6), and the non-porous NIP film (5).

the MIP film with interconnecting channels, prepared using silica colloidal crystals, similarly to what has been observed for other sensors based on porous metals. ${ }^{47,48}$
Table 1 Analytical parameters of the MIP film chemosensors for L-arabitol

\begin{tabular}{lll}
$\begin{array}{l}\text { Chemosensor } \\
\text { recognition unit }\end{array}$ & Analytical parameter & Value \pm st. dev. \\
\hline $\begin{array}{l}\text { Non-porous } \\
\text { (L-arabitol)-MIP }\end{array}$ & $\begin{array}{l}\text { Sensitivity to L-arabitol } \\
\text { analyte, Hz } \mathrm{mM}^{-1} \\
\text { Linear dynamic concentration } \\
\text { range, mM }\end{array}$ & $-19.78 \pm 0.53$ \\
& $\begin{array}{l}\text { Limit of quantitation, } \mathrm{mM} \\
\text { Sensitivity to interferences, }\end{array}$ & 0.150 \\
& $\begin{array}{l}\text { Hz } \mathrm{mM}^{-1} \\
\text { D-Arabitol }\end{array}$ & $-0.88 \pm 0.82$ \\
& $\begin{array}{l}\text { Xylitol } \\
\text { Ribitol }\end{array}$ & $-1.42 \pm 0.97$ \\
& $\begin{array}{l}\text { Sensitivity to L-arabitol analyte, } \\
\text { Hz mM }\end{array}$ & $-36.51 \pm 1.27$ \\
$\begin{array}{l}\text { Macroporous } \\
\text { (L-arabitol)-MIP }\end{array}$ & $\begin{array}{l}\text { Linear dynamic concentration } \\
\text { film }\end{array}$ & $0.009-1.250$ \\
& Limit of quatitation, $\mu \mathrm{M}$ & 9 \\
Non-porous & $\begin{array}{l}\text { Sensitivity to L-arabitol, } \\
\text { NIP film }\end{array}$ & Hz mM ${ }^{-1}$
\end{tabular}

The film macroporosity facilitated binding of $\mathrm{L}$-arabitol molecules to MIP molecular cavities by enhancing their diffusion deeper into the film. Interestingly, the non-porous and macroporous film reached binding saturation at a similar time (Fig. 4b). However, higher and stable binding of L-arabitol was not only evident by studying the time dependence of the association, but also by following the dissociation with time. Approximately twice as much time was necessary to extract the analyte completely (dissociation of the covalent bond formed between L-arabitol and boronic acid) in comparison to the non-porous MIP film (Fig. 4b). The LOD for the macroporous chemosensor was sufficient to determine $\mathrm{L}$-arabitol at concentrations typical for blood plasma, i.e., in the micromolar range. Table 1 summarizes the comparative analytical parameters of these two MIP chemosensors.

The presence of selective molecular cavities imprinted in the polymer was confirmed by determining the imprinting factor. This factor is defined by the ratio of the sensitivity of the MIP chemosensor $\left(-19.78 \mathrm{~Hz} \mathrm{mM}^{-1}\right)$ (Table 1) to that of the NIP chemosensor $\left(-4.21 \mathrm{~Hz} \mathrm{mM}^{-1}\right.$ ) (Table 1). This ratio was as high as $\sim 4.5$. Such a high value indicates a homogenous recognition site distribution in the MIP matrix and confirms suitability of the functional monomer. Moreover, selectivity of the chemosensor for L-arabitol was determined as the ratio of sensitivity to the L-arabitol analyte and to potentially interfering compounds including D-arabitol, xylitol, and ribitol (Fig. 4c and Table 1). Advantageously, the chemosensor did not respond to xylitol and ribitol, close structural analogs of L-arabitol. Moreover, it was completely insensitive to D-arabitol, an enantiomer of opposite chirality.

\section{Conclusions}

A relatively simple and inexpensive procedure was developed for the fabrication of an MIP chemosensor for the selective determination of $\mathrm{L}$-arabitol. The chemosensor revealed very significant enantio- and stereoselectivity with respect to other 
sugar alcohols. Colloidal crystal templating for increasing the specific surface area of the MIP film substantially improved the detectability to reach concentrations comparable to those typical found for L-arabitol in blood plasma. This procedure of L-arabitol determination is an attractive alternative for the currently used clinical determination procedures. We anticipate that the herein developed protocol of molecular imprinting has the potential of use in practical clinical applications for the determination of the $\mathrm{D}^{-}$to L-arabitol ratio in body fluids and, therefore, opens the door to early diagnosis of fungal infections. Furthermore, this protocol can easily be adapted for other types of analytes and, therefore, might help to improve the performance of several other electrochemical and piezomicrogravimetric chemosensors.

\section{Acknowledgements}

The present research was funded by the Polish National Science Centre (NCN) through Grant No. 2011/N/ST4/03491 to M. D. Moreover, the authors acknowledge NCN (Grant No. 2011/03/D/ ST4/02596 to P. S. S.), the European Regional Development Fund (Grant No. POIG.01.01.02-00-008/08 to P. S. S., K. N. and W. K.), and the European $7^{\text {th }}$ Framework Programme (Grant No. FP7-REGPOT-CT-2011-285949-NOBLESSE to W. K.) for financial support.

\section{References}

1 P. T. Kissinger, Biosens. Bioelectron., 2005, 20, 2512-2516.

2 P. D’Orazio, Clin. Chim. Acta, 2003, 334, 41-69.

3 Y. H. Lau, P. J. Rutledge, M. Watkinson and M. H. Todd, Chem. Soc. Rev., 2011, 40, 2848-2866.

4 L. E. Santos-Figueroa, M. E. Moragues, E. Climent, A. Agostini, R. Martınez-Manez and F. Sancenon, Chem. Soc. Rev., 2013, 42, 3489-3613.

5 L. You, D. Zha and E. V. Anslyn, Chem. Rev., 2015, 115, 7840-7892.

6 J. F. Teichert, D. Mazunin and J. W. Bode, J. Am. Chem. Soc., 2013, 135, 11314-11321.

7 F. L. Dickert, P. Lieberzeit, S. G. Miarecka, K. J. Mann, O. Hayden and C. Palfinger, Biosens. Bioelectron., 2004, 20, 1040-1044.

8 J. Wackerlig and R. Schirhagl, Anal. Chem., 2016, 88, 250-261.

9 A. Speltini, A. Scalabrini, F. Maraschi, M. Sturini and A. Profumo, Anal. Chim. Acta, 2017, 974, 1-26.

10 E. L. Holthoff and F. V. Bright, Anal. Chim. Acta, 2007, 594, 147-161.

11 P. S. Sharma, M. Dabrowski, F. D'Souza and W. Kutner, TrAC, Trends Anal. Chem., 2013, 51, 146-157.

12 L. Li, L. Yang, Z. Xing, X. Lu and X. Kan, Analyst, 2013, 138, 6962-6968.

13 R. B. Pernites, S. K. Venkata, B. D. B. Tiu, A. C. C. Yago and a. R. C. Advincula, Small, 2012, 8, 1669-1674.

14 B. D. B. Tiu, S. B. Tiu, A. M. Wen, P. Lam, N. F. Steinmetz and R. C. Advincula, Langmuir, 2016, 32, 6185-6193.
15 B. D. B. Tiu, R. B. Pernites, S. B. Tiu and R. C. Advincula, Colloids Surf., A, 2016, 495, 149-158.

16 J. C. Yang and J. Y. Park, ACS Appl. Mater. Interfaces, 2016, 8, 7381-7389.

17 S. Reculusa, M. Heim, F. Gao, N. Mano, S. Ravaine and A. Kuhn, Adv. Funct. Mater., 2011, 21, 691-698.

18 R. I. Boysen, L. J. Schwarz, D. V. Nicolau and M. T. W. Hearn, J. Sep. Sci., 2017, 40, 314-335.

19 B. P. Guery, M. C. Arendrup, G. Auzinger, E. Azoulay, M. B. Sa, E. M. Johnson, E. Muller, C. Putensen, C. Rotstein, G. Sganga, M. Venditti, R. Z. Crespo and B. J. Kullberg, Intensive Care Med., 2009, 35, 55-62.

20 M. A. Johnson and D. R. Bundle, Chem. Soc. Rev., 2013, 42, 4327-4344.

21 L. Alcazar-Fuoli and E. Mellado, Br. J. Haematol., 2014, 166, 471-484.

22 B. P. Guery, M. C. Arendrup, G. Auzinger, E. Azoulay, M. B. Sa, E. M. Johnson, E. Muller, C. Putensen, C. Rotstein, G. Sganga, M. Venditti, R. Z. Crespo and B. J. Kullberg, Intensive Care Med., 2009, 35, 206-214.

23 L. Lehtonen, V.-J. Anttila, T. Ruutu, J. Salonen, J. Nikoskelainen, E. Eerola and P. Ruutu, J. Clin. Microbiol., 1996, 2175-2179.

24 G. Sigmundsdottir, B. Christensson, L. J. Bjorklund, K. Hakansson, C. Pehrson and L. Larsson, J. Clin. Microbiol., 2000, 3039-3042.

25 J. H. Huck, N. M. Verhoeven, E. A. Struys, G. S. Salomons, C. Jakobs and M. S. van der Knaap, Am. J. Hum. Genet., 2004, 74, 745-751.

26 M. C. Arendrup, O. J. Bergmann, L. Larsson, H. V. Nielsen, J. O. Jarløv and B. Christensson, Clin. Microbiol. Infect., 2010, 16, 855 .

27 L. Larsson, C. Pehrson, T. Wiebe and B. Christensson, J. Clin. Microbiol., 1994, 32, 1855-1859.

28 M. Dabrowski, P. S. Sharma, Z. Iskierko, K. Noworyta, M. Cieplak, W. Lisowski, S. Oborska, A. Kuhn and W. Kutner, Biosens. Bioelectron., 2016, 79, 627-635.

29 R.-J. Bensadoun, L. L. Patton, R. V. Lalla and J. B. Epstein, Support Care Cancer, 2011, 19, 737-744.

30 T. Kiehn, E. Bernard, J. Gold and D. Armstrong, Science, 1979, 206, 577-580.

31 L. Stobinski, L. Zommer, I. Flis-Kabulska, M. Mecina, B. Gniewinska, W. Szulc and W. Kutner, J. Electrochem. Soc., 2000, 147, 4203-4205.

32 S. Reculusa, C. Poncet-Legrand, S. Ravaine, C. Mingotaud, E. Duguet and E. Bourgeat-Lami, Chem. Mater., 2002, 14, 2354-2359.

33 S. Reculusa and S. Ravaine, Appl. Surf. Sci., 2005, 246, 409-414.

34 S. Reculusa and S. Ravaine, Chem. Mater., 2003, 15, 598-605.

35 S. Reculusa, P. Massé and S. Ravaine, J. Colloid Interface Sci., 2004, 279, 471-478.

36 B. v. Duffel, R. H. A. Ras, F. C. D. Schryver and R. A. Schoonheydt, J. Mater. Chem., 2001, 11, 3333-3336.

37 M. Heim, S. Reculusa, S. Ravaine and A. Kuhn, Adv. Funct. Mater., 2012, 22, 538-545. 
38 A. Kochman, A. Krupka, J. Grissbach, W. Kutner, B. Gniewinska and L. Nafalski, Electroanalysis, 2006, 18, 2168-2173.

39 K. Karim, F. Breton, R. Rouillon, E. V. Piletska, A. Guerreiro, I. Chianella and S. A. Piletsky, Adv. Drug Delivery Rev., 2005, 57, 1795-1808.

40 X. Wu, Z. Li, X.-X. Chen, J. S. Fossey, T. D. James and Y.-B. Jiang, Chem. Soc. Rev., 2013, 42, 8032-8048.

41 P. N. Bartlett, P. R. Birkina and M. A. Ghanem, Chem. Commun., 2000, 1671-1672.

42 J. Heinze, B. A. Frontana-Uribe and S. Ludwigs, Chem. Rev., 2010, 110, 4724-4771.

43 P. N. Bartlett, P. R. Birkin, M. A. Ghanema and C.-S. Toh, J. Mater. Chem., 2001, 11, 849-853.
44 A. Pietrzyk, W. Kutner, R. Chitta, M. E. Zandler, F. D’Souza, F. Sannicolo and P. R. Mussini, Anal. Chem., 2009, 81, 10061-10070.

45 A. Pietrzyk, S. Suriyanarayanan, W. Kutner, R. Chitta, M. E. Zandler and F. D'Souza, Biosens. Bioelectron., 2010, 25, 2522-2529.

46 D. A. Buttry and M. D. Ward, Chem. Rev., 1992, 92, 1355-1379.

47 T. Yutthalekha, C. Wattanakit, V. Lapeyre, S. Nokbin, C. Warakulwit, J. Limtrakul and A. Kuhn, Nat. Commun., 2016, 7, 12678.

48 C. Wattanakit, Y. B. S. Come, V. Lapeyre, P. A. Bopp, M. Heim, S. Yadnum, S. Nokbin, C. Warakulwit, J. Limtrakul and A. Kuhn, Nat. Commun., 2014, 5, 3325. 